\title{
Osteosarcoma amplified 9 is highly expressed in mouse adipocytes and controls lipid storage
}

\author{
LUXIN YU $^{1}$, ZONGQIAN NIAN $^{2}$ and JIANLI SANG ${ }^{1}$ \\ ${ }^{1}$ Key Laboratory for Cell Proliferation and Regulation Biology of Ministry of Education, Institute of Cell Biology, \\ College of Life Sciences, Beijing Normal University; ${ }^{2}$ Protein Science Laboratory of Ministry of Education, \\ School of Life Sciences, Tsinghua University, Beijing, P.R. China
}

Received January 4, 2011; Accepted April 14, 2011

DOI: $10.3892 / \mathrm{mmr} .2011 .480$

\begin{abstract}
Adipocytes are among the most important cells for energy storage, metabolism and balance in mammals. Dysfunctional adipocytes are closely associated with type 2 diabetes and obesity, and thus with insulin resistance and inflammation. Recent studies show that endoplasmic reticulum (ER) stress may be responsible for obesityinduced insulin resistance. In this study, we investigated an ER-associated degradation-related protein in adipocytes, namely osteosarcoma amplified 9 (OS-9), a protein that is reported to have lectin activity and to be involved in ER quality control. We found that OS-9 is expressed at high levels in both types of fat tissues - brown and white adipose tissues - and we examined the subcellular localization of OS-9 in 3T3-L1 adipocytes. Furthermore, we observed that the expression levels of the OS-9 protein gradually increased as 3T3-L1 adipocytes differentiated. Most notably, we found that the depletion of this protein influences adipocyte differentiation and lipid storage, although the mechanism behind this is unclear.
\end{abstract}

\section{Introduction}

Adipocytes and adipose tissue (which is mainly composed of adipocytes) are highly specialized for energy metabolism. Their functions include storage and release in response to a variety of environmental signals. Alteration of white adipose

Correspondence to: Dr Jianli Sang, Key Laboratory for Cell Proliferation and Regulation Biology of Ministry of Education, Institute of Cell Biology, College of Life Sciences, Beijing Normal University, Beijing 100875, P.R. China

E-mail: jlsang@bnu.edu.cn

Abbreviations: OS-9, osteosarcoma amplified 9; ER, endoplasmic reticulum; ERAD, ER-associated degradation; FABP, adipocytespecific fatty acid transporting protein; TAG, triacylglycerol; siRNA, small interference RNA; GFP, green fluorescent protein

Key words: osteosarcoma amplified 9, adipocyte, lipid storage, differentiation, endoplasmic reticulum stress tissue by dysfunctional expression and secretion of adipokines leads to obesity, diabetes and other metabolic diseases (1). In recent years, endoplasmic reticulum (ER) stress has been reported to play a crucial role in the obesity-induced insulin resistance found in type 2 diabetes $(2,3)$. Several stress-related proteins, such as oxygen-regulated protein 150 and c-Jun amino-terminal kinase, are elevated in obesity, and these contribute directly to the impairment of insulin sensitivity and insulin receptor signaling capacity (2-5).

YOS9, the putative yeast homolog of a gene amplified in osteosarcomas, was originally identified in Saccharomyces cerevisiae as being responsible for targeting certain incorrectly folded glycosylated proteins, such as CPY, to the ubiquitin conjugation system for ER-associated degradation (ERAD) (6-8). YOS9 is recruited into a protein complex containing Hrd1P ubiquitin ligase; Kar2p, an ER lumenal chaperone; and Hrd3p, a lumenally exposed transmembrane tetratricopeptide repeat-containing protein (8-10). Osteosarcoma amplified 9 (OS-9), which was originally identified by its up-regulation in osteosarcoma (11) and myeloid leukemia (12), shares limited homology with YOS9 (13). OS-9 has been found to interact with several proteins, including hypoxia-inducible factor $1 \alpha(\mathrm{HIF} 1 \alpha)$ and prolyl hydroxylases, to promote the oxygen-dependent degradation of HIF1 $\alpha$ (14). OS-9 was recently shown to be an ER-residing lectin that binds to ERAD substrates and to the membrane-embedded Hrd1-Sel1L ubiquitin ligase complex to coordinate substrate recognition in the ER lumen with ubiquitin conjugation in the cytoplasm (13). In addition, it has been reported that OS-9 has the ability to bind sugar (15), and its lectin activity has been reported to be required in glycoprotein ERAD (16). OS-9 is closely related to ER stress, not only because the ERAD pathway is the primary mechanism responsible for degrading the misfolded proteins that cause ER stress, but also because it is up-regulated significantly in response to ER stress $(17,18)$. Here, we observed that OS-9 had high expression levels in adipocytes, and that its expression level gradually increased as the adipocytes differentiated. Furthermore, the depletion of OS-9 in 3T3-L1 adipocytes impaired cell function in terms of triacylglycerol (TAG) storage, and slightly influenced cell differentiation. Thus, it can be concluded that OS-9 plays an important role in adipocyte energy storage, although the exact mechanism behind this requires further investigation. 


\section{Materials and methods}

Plasmids and antibodies. The siRNA-expressing vector pSilencer2.1-U6-Hygro was purchased from Ambion; the lentiviral vector FG12 and the packaging vectors pMDLg/pRRE, pREV-Rev and pVSVG were gifts from Dr Zilong Wen (Hong Kong University of Science and Technology). Full-length and truncated mouse OS-9 isoforms (1 and 2) were amplified from mouse white adipose tissue cDNA using PCR and inserted into the pCMV5-HA, pcDNA3 or pET28a vectors. All constructs were verified by DNA sequencing.

Antibody against HA was purchased from Santa Cruz Biotechnology (Santa Cruz, CA, USA), antibody against FABP from ADI, antibodies against $\beta$-actin and CNX from Sigma (USA) and antibody against ADRP from Fitzgerald Industries. Tunicamycin (TN), thapsigargin (TG), phenylmethylsulfonyl (PMSF), leupeptin, $\beta$-glycerol phosphate, sodium orthovandate and sodium pyrophosphate were all purchased from Sigma.

Generating a polyclonal antibody against two isoforms of $O S$-9. A single colony of E. coli strain BL21 (DE3) harboring pET28a-OS9 (amino acid 326-672) was amplified for $6 \mathrm{~h}$ and $0.2 \mathrm{mM}$ IPTG was added into the medium to induce protein expression for $6 \mathrm{~h}$. The cells were then harvested and lysed by mild sonication. The lysate was subjected to centrifugation at 12,000 rpm for $15 \mathrm{~min}$. Pellets were discarded and the supernatant was incubated on a Ni-column at $4^{\circ} \mathrm{C}$ for $1 \mathrm{~h}$ to enrich the OS-9 C-terminal protein. The protein was then eluted from the column and the purity was evaluated using SDS-PAGE gel stained with coomassie brilliant blue.

Mice older than 2 months were selected to generate antiserum. Complete and incomplete Freund's adjuvants were mixed with the antigen. Two months after injection, whole blood was generated and serum was separated.

Next, $400 \mu \mathrm{g}$ purified OS-9 C-terminal protein was subjected to SDS-PAGE, and the proteins were transferred to a PVDF membrane. The membrane was stained with $0.5 \%$ Ponceau S. for $5 \mathrm{~min}$, and a section of membrane, chosen according to the molecular weight of the protein, was incubated overnight with the serum (derived as described above). Finally, the polyclonal antibody against mouse OS-9 was eluted from the membrane using $600 \mu l$ glycine ( $\mathrm{pH} 2.5$ ).

Cell culture and transfection. HEK293T and 3T3-L1 cells were maintained in DMEM (Gibco, USA) containing 10\% fetal bovine serum (FBS; Gibco). The differentiation of the 3T3-L1 cells was initiated 2 days post-confluence by the addition of $5 \mu \mathrm{g} / \mathrm{ml}$ insulin, $1 \mu \mathrm{M}$ dexamethasone (DEX) and $0.5 \mathrm{mM}$ isobutylmethylxanthine (IBMX) (Sigma), and the reaction was allowed to proceed for two days. The differentiation medium was subsequently replaced with DMEM/FBS supplemented only with $5 \mu \mathrm{g} / \mathrm{ml}$ insulin for two more days, which was then replaced with normal culture medium.

$293 \mathrm{~T}$ cells were transfected using the previously described calcium-phosphate co-precipitation technique (19) or Lipofectamine 2000 (Invitrogen, USA) according to the manufacturer's instructions.

Virus preparation. siRNA against OS-9 (GTACAAACA GCGCTATGAGT) was cloned into the FG12 expression vector and packaged into lentivirus as previously described $(20,21)$. 3T3-L1 preadipocytes were infected for $12 \mathrm{~h}$ with the lentivirus expressing the OS-9-specific siRNA. After several passages, infected preadipocytes that stably expressed the siRNA were used as an OS-9 knockdown cell line, and differentiation was induced. Since all lentiviruses contain a GFP expression unit, GFP was used as the control for viral infection efficiency. A lentivirus generated from the empty vector, which expressed only GFP, was used as the negative control.

Measurement of TAG content. TAG of 3T3-L1 adipocytes was extracted and calculated as previously described $(21,22)$.

Subcellular fractionation. This method for evaluating OS-9 localization was essentially the same as described previously (23).

\section{Results}

OS-9 is highly expressed in adipocytes from adipose tissue and liver. To investigate OS-9 expression in tissues and cells as well as to learn more about its function, an OS-9 polyclonal antibody was prepared from mice. Mouse OS-9 truncated protein (aa 326-672) was expressed and purified, and the protein was injected into mice to trigger an immune response. After the mice were immunized for 2 months, the serum was collected and used for evaluating recognition efficiency. As shown in Fig. 1A, serum samples 1,2 and 3 specifically recognized two isoforms of OS-9 proteins ectopically expressed in 293T cells. Serum 1 was further purified using the truncated OS-9 protein (aa 326-672) by affinity purification.

Although OS-9 is known to be ubiquitously expressed in mammalian cells, the expression patterns of OS-9 in adipocytes have not yet been characterized. To verify the OS-9 expression levels in fat cells, brown adipose tissue (BAT), white adipose tissue (WAT) and liver were isolated from wild-type mice. Total RNA was extracted and reverse-transcribed into cDNA using Oligo dT. Specific primers for OS-9 were applied to amplify templates and examine mRNA levels. It was observed that OS-9 mRNA levels are very high in three types of fat cells. The protein levels of OS- 9 isoforms 1 and 2 detected by the OS-9 antibody were both consistently high in these fat cells, as shown in Fig. 1B.

OS-9 is localized on endoplasmic reticulum in adipocytes. Several studies conducted in recent years have indicated that OS-9 resides on the ER. However, the precise subcellular localization of this protein in adipocytes has not yet been investigated. Since adipocytes have a large number of lipid droplets - key organelles required for adipocytes' biological functions - we sought to determine whether OS-9 was localized to these organelles. Subcellular biochemical fractionation was carried out using sucrose gradient centrifugation. Organelles were separated into four fractions on the basis of density: lipid droplets, S100 cytosol, high-density microsomes and low-density microsomes (23). By monitoring different organelle marker proteins, the purity of each fraction was evaluated. $\beta$-actin was detected only in the cytosolic fraction, whereas calnexin, an ER-specific protein, was observed at a high level in both high- and low-density microsomes. 
A

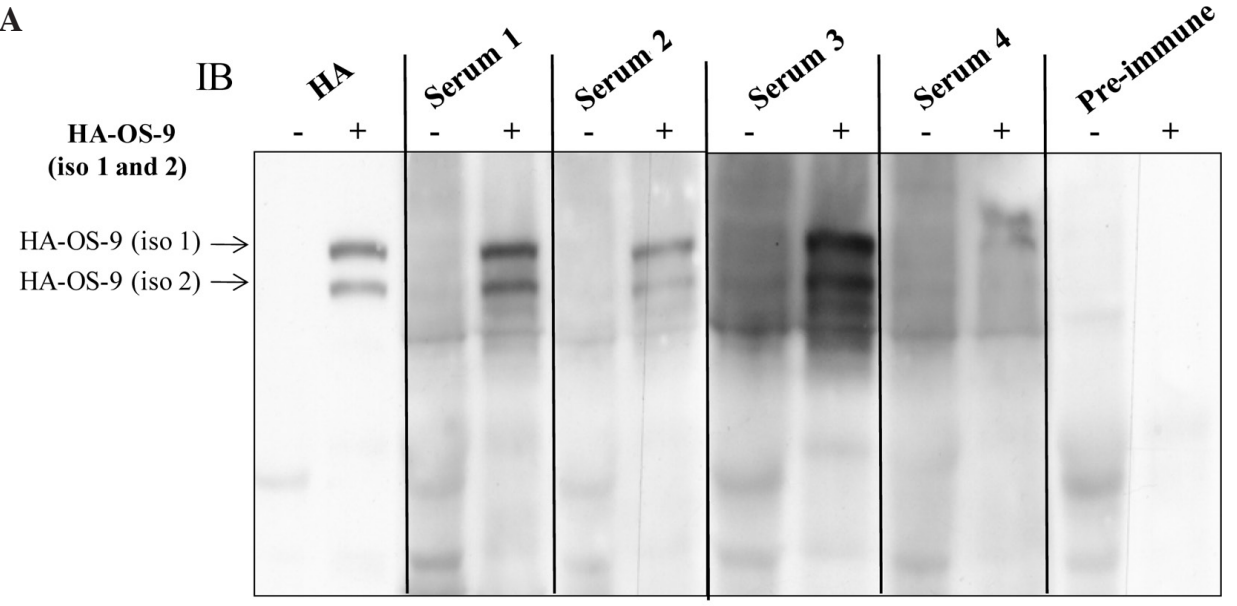

B

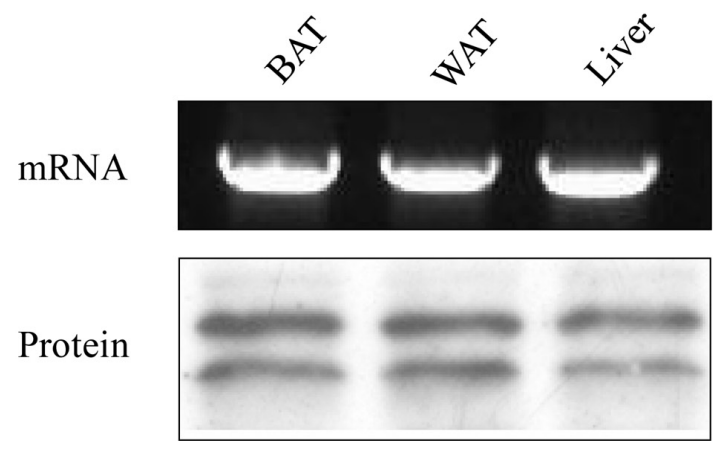

Figure 1. OS-9 is expressed in adipose tissues. (A) Recognition efficiency of anti-sera. Truncated OS-9 protein (326-672) was purified and injected into mice for anti-serum. Sera from immunized mice were isolated. Plasmids encoding HA-tagged OS-9 isoforms 1 and 2 (HA-OS-9 iso 1 and iso 2 ) were overexpressed in 293T cells. Twenty-four hours later, cells were harvested for the detection of the OS-9 protein by applying the collected sera. (B) Expression of OS-9 in adipose tissues. Protein and total RNA samples of adipose tissue from adult wild-type mice were acquired to examine OS-9 protein and mRNA levels. Endogenous OS-9 was recognized using the polyclonal antibody purified from serum 1 in (A).

Lipid droplets, a representative organelle in adipocytes, have only ADRP, with no other contaminants. The data in Fig. 2 clearly show that the OS-9 protein co-fractionated with the ER-specific marker calnexin, and was enriched in both highand low-density microsomes. It was absent in the lipid droplet fraction and the cytosol fraction.

The expression level of OS-9 gradually increases during adipocyte differentiation. The protein expression profile of adipocytes is significantly different from that of other cell types, which is consistent with the fact that adipocytes execute special tasks, such as storing large amounts of fuels and metabolizing energy to adapt to different environmental conditions. When fibroblasts are induced to differentiate into adipocytes, many events occur that allow adipogenic genes to become active, creating functional adipocytes. Since OS-9 has a high expression level in adipose tissues, we sought to determine whether OS-9 expression levels change during the transition from fibroblasts to adipocytes. To monitor this process, 3T3-L1 preadipocytes (fibroblasts) were cultured and induced to differentiate. Generally, cells were considered to be mature adipocytes on day 8 after induction. During the differentiation process, cells were harvested at 2-day intervals and protein expression levels were evaluated using Western blotting. As shown in Fig. 3A, OS-9 protein levels increased gradually as cells transitioned from fibroblasts to adipocytes. Notably, the expression reached a peak on day 8 , the same stage at which

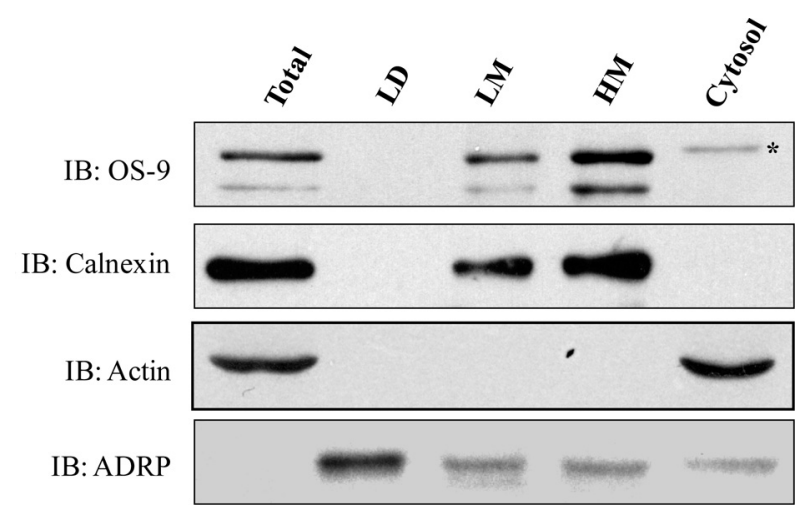

Figure 2. Subcellular localization of OS-9 in 3T3-L1 adipocytes. 3T3-L1 fibroblasts were induced to differentiate into adipocytes. On day 8 after induction for differentiation, subcellular fractions were isolated as mentioned in Materials and methods. Calnexin, $\beta$-actin and ADRP were applied as markers for microsome, cytosol and lipid droplet fractions, respectively. LD, lipid droplets; LM, low-density microsomes; HM, high-density microsomes. "Non-specific band.

the expression of the adipocyte-specific fatty acid transporting protein (FABP), a differentiation marker protein for adipocytes, peaked. $\beta$-actin was applied as an internal control to normalize cell amount. In addition, total RNA from cells on days 2 and 8 was isolated and reverse-transcribed into cDNA for OS-9 mRNA analysis. The mRNA levels of OS-9 in mature 
A

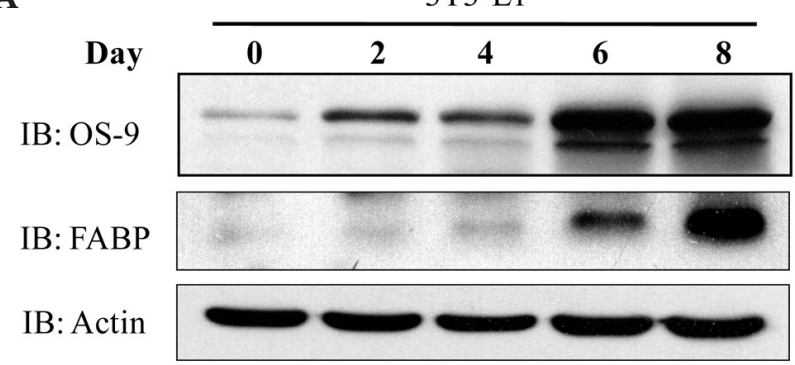

B

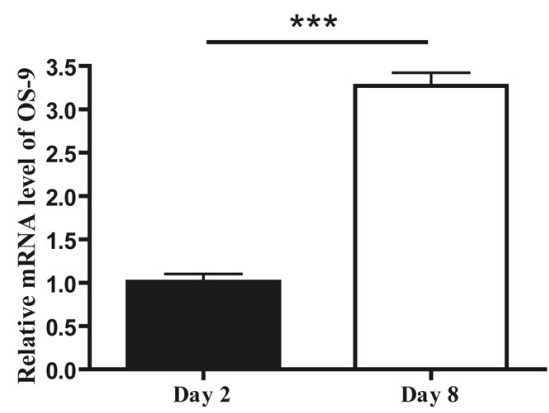

C

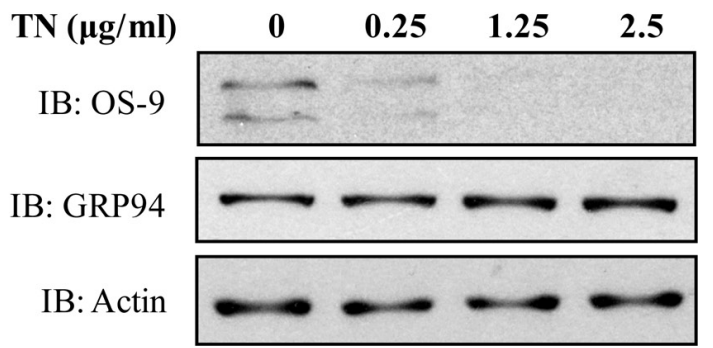

Figure 3. Protein expression profile of OS-9 in 3T3-L1 adipocytes. (A) OS-9 expression during the adipocyte differentiation. Protein expression levels of OS-9 were examined during the process of cell differentiation from fibroblasts to mature adipocytes. Cells were harvested on days $0,2,4,6$ and 8 after being induced for differentiation. (B) Comparison of OS-9 mRNA levels in adipocytes during the differentiation process. Quantitative real-time PCR analysis was used to analyze the OS-9 mRNA levels. The relative mRNA level of OS-9 on day 2 was designated as 1 . (**** $\mathrm{p}<0.001, \mathrm{n}=4)$. (C) Alteration of OS-9 expression in response to ER stress. Differentiated 3T3-L1 adipocytes were treated with TN $(0.25,1.25$ and $2.5 \mu \mathrm{g} / \mathrm{ml})$ or DMSO for $24 \mathrm{~h}$ and cells were harvested for the detection of endogenous OS-9 and GRP94 protein levels.

adipocytes were much higher than those of cells on day 2 after induction of differentiation ( 2-fold higher; Fig. 3B). FABP has been reported to play an important role in adipogenesis and fatty acid transportation in adipocytes (24). The fact that OS-9 has a similar expression pattern and an adipogenic-like gene transcriptional pattern suggests that OS-9 may also be involved in adipocyte function.

OS-9 protein level is down-regulated in response to ER stress in adipocytes. Previous studies have reported that OS-9 participates in the ERAD pathway, and that its protein levels are increased when ER stress is induced (18). In this study, tunicamycin (TN) and thapsigargin (TG) were applied to induce ER stress in adipocytes and changes in OS-9 protein levels were observed. Unexpectedly, OS- 9 was found to respond to ER stress, but its levels decreased significantly after TN

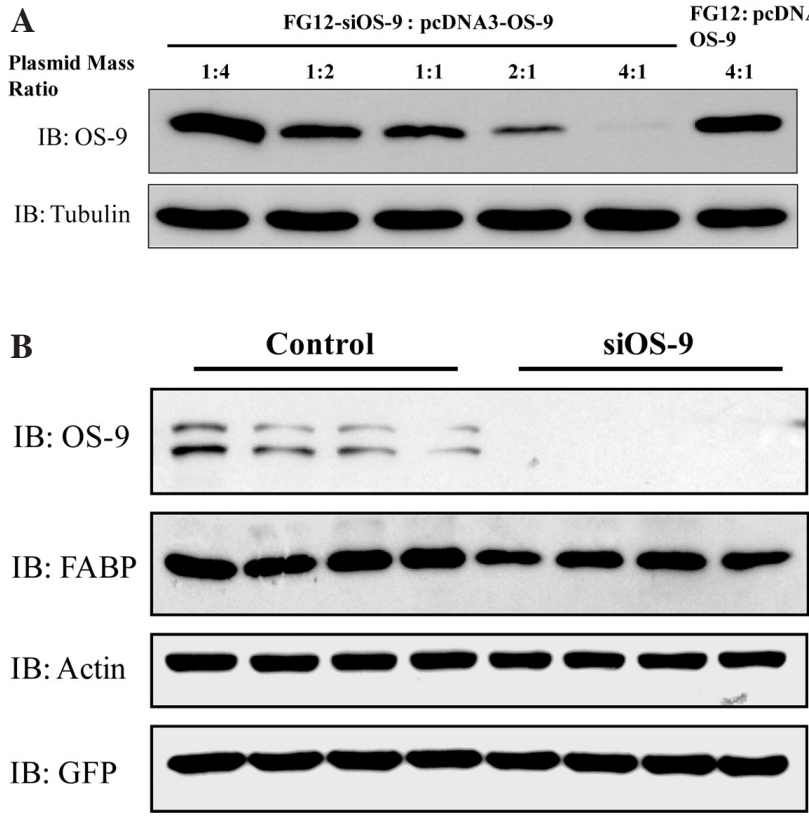

$\mathbf{C}$

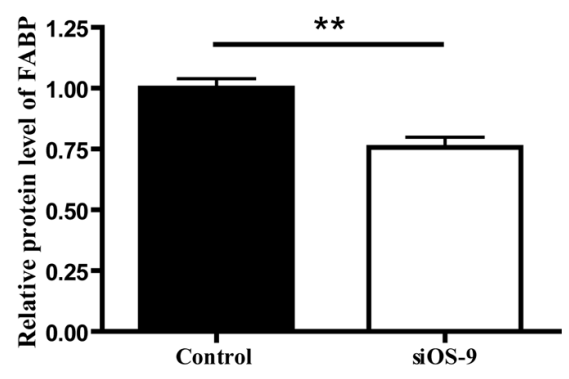

D

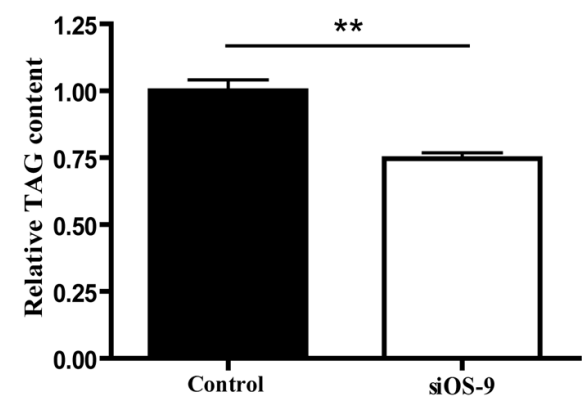

Figure 4. Protein expression and TAG content in 3T3-L1 adipocytes with an OS-9 deficiency. (A) Efficiency of siRNA specific for silencing OS-9. Different ratios of FG12 vector encoding OS-9 siRNA to pcDNA3 vector expressing OS-9 isoform 1 were transfected into $293 \mathrm{~T}$ cells. At $24 \mathrm{~h}$ after transfection, cells were harvested to analyze the OS-9 protein levels. (B) Generation of OS-9-deficient 3T3-L1 cells. 3T3-L1 fibroblasts infected by a lentivirus encoding a siRNA specific for OS-9 mRNA were generated and induced to differentiate. Cells on day 8 after induction for differentiation were harvested to analyze OS-9 and FABP protein levels. (C) Quantitative analysis of Western blotting in Fig. 4B. ( $\left.{ }^{*} \mathrm{p}<0.01, \mathrm{n}=4\right)$. (D) Relative TAG content in OS-9-deficient adipocytes. On day 8 after 3T3-L1 fibroblasts were induced for differentiation, total lipids of cells were extracted and separated by TLC. TAG content was calculated using Quantity One software. The relative TAG level of wild-type adipocytes was designated as $1 .\left({ }^{* *} \mathrm{p}<0.001, \mathrm{n}=3\right)$.

treatment (Fig. 3C) and TG treatment (data not shown), while the GRP94 level increased. This observation differed from patterns observed in other cell types. Although the reason for this is unclear, these findings suggest that the function of OS-9 in adipocytes may differ from its function in other cell types. 
Adipocyte-specific fatty acid transporting protein levels are slightly decreased in OS-9 knockdown adipocytes. To further investigate whether OS-9 is involved in adipocyte function, siRNAs were designed specific for both isoforms 1 and 2 of OS-9 mRNA and cloned into the RNA expression vector FG12. In the HEK293T cells, the silencing efficiency of the siRNA was examined by co-transfecting the OS-9 isoform 1 expression vector with this FG12 construct. It was observed that the knockdown efficiency increased as the ratio of the FG12 vector harboring OS-9 siRNA to the OS-9 expression vector increased, which indicates an excellent silencing effect of the siRNA expressed by FG12 (Fig. 4A). Next, FG12 was transfected into the 293T cells with the other expression vectors required to package the lentivirus. 3T3-L1 preadipocytes were fully infected with this lentivirus to generate cells that stably express siRNA for OS-9. A lentivirus expressing only GFP, which was packaged by transfecting the empty FG12 vector, was applied as the negative control.

After a few generations of selection, 3T3-L1 fibroblasts were cultured to differentiate into 3T3-L1 adipocytes. On day 8 after the induction of differentiation, the expression levels of endogenous OS-9 and FABP proteins were analyzed. The consistency of $\beta$-actin and GFP protein levels indicated a similar cell number and an efficient lentivirus infection, respectively. As shown in Fig. 4B, the OS-9 protein level was significantly reduced, as expected. Meanwhile, FABP levels were decreased by $\sim 25 \%$ in the OS-9-deficient adipocytes compared to the wild-type adipocytes (Fig. 4B and C), which suggests that the characteristics and the differentiation ability of the adipocytes were affected after silencing of OS-9.

Lipid storage of adipocytes is reduced after OS-9 silencing. To further investigate whether OS-9 influences the function of adipocytes, lipid storage - the most prominent function of adipocytes - was examined after the silencing of OS-9 expression. On day 8 after 3T3-L1 fibroblasts that constitutively express siRNA specific for OS-9 were induced for differentiation, total lipids of these cells were isolated and then separated by thin-layer chromatography. Finally, the TAG content was measured. Strikingly, the levels of TAG, the primary lipid form of adipocytes, were reduced in OS-9-deficient adipocytes by $\sim 30 \%$ compared to the wild-type adipocytes (Fig. 4D). This observation suggests that OS-9 regulates the lipid storage of adipocytes in some specific processes. However, the detailed molecular mechanism requires further investigation.

Through various analyses, we verified that OS-9 is expressed in adipose tissue and adipocytes, and that it localizes to ER in adipocytes. Furthermore, OS-9 expression profiles were examined during the process of adipocyte differentiation. Most notably, we observed that OS-9 deficiency in fat cells influences cell differentiation and their main function, namely, lipid storage. Together, these data indicate that OS-9 is closely related to adipocyte differentiation and function.

\section{Discussion}

OS-9 has been reported to play an essential role in the ERAD pathway. It is responsible for recognizing misfolded proteins and delivering these proteins to E3-ubiquitin ligase for degradation. However, OS-9 has also been shown to specifically protect some proteins from degradation before maturation, ensuring their biological functionality (25). Together, these findings suggest that OS-9 plays a role in the regulation of protein degradation. Since it is involved in protein quality control, it is closely related to ER stress. There have also been reports that OS-9 protein levels increase after ER stress is induced.

In additional research on OS-9 in adipocytes, we observed that OS-9 also responds to ER stress in adipocytes. However, its protein levels were found to be significantly decreased rather than increased after ER stress was induced, which suggests that it may act differently in adipocytes as compared to other cell types. To further investigate its functions in adipocytes, a polyclonal antibody was prepared, specific for OS-9 isoforms 1 and 2, and applied to detect OS-9 protein levels during the adipocyte differentiation process. We found that there is a basal expression of OS-9 in fibroblasts and that its expression level gradually increased as the 3T3-L1 fibroblasts differentiated to adipocytes. The expression profile is similar to that of the differentiation marker FABP, which reached a peak level when 3T3-L1 cells developed into mature adipocytes. Since the function of adipocytes is closely related to their differentiation, their function is fully acquired only when cells become mature adipocytes. Thus, OS-9 may participate in helping to maintain or execute adipocyte function, distinct from its function in fibroblasts.

To confirm this hypothesis, a stable cell line with depleted OS- 9 was constructed and then differentiated into adipocytes. As shown by the results, the levels of both OS-9 protein and the adipocyte differentiation marker FABP were attenuated. This phenotype indicates that the differentiation extent of adipocytes was affected, and that cell function may also be influenced. The decrease of TAG content in adipocytes with depleted OS-9 confirmed this hypothesis. It is possible that the differentiation of adipocytes was affected so that the function could not be fully acquired. Since FABP is involved in adipocyte function, another possibility is that the function of the adipocytes was impaired so that they appeared to have a phenotype that is not fully differentiated. Regardless, it is certain that both the differentiation and the function of adipocytes were weakened after OS-9 protein levels were reduced.

The results of subcellular fractionation may shed some light on how OS-9 influences the function and differentiation of adipocytes. It was shown that OS-9 localizes only to the ER and not to the nucleus (data not shown) or the cytosol. This indicates that OS-9 has a very low likelihood of being a transcription regulator. In addition, OS-9 is not targeted to lipid droplets, indicating that it does not directly promote TAG storage, in terms of affecting lipid droplet growth or fusion. Thus, its regulation may be related to its action of protein degradation control, as shown in previous studies. However, OS-9 cannot directly interact with FABP (data not shown), so FABP is not the direct effector of OS-9. The direct effector controlled by OS-9 is possibly an upstream regulator of FABP. OS-9 may control the degradation of this effector(s), thereby contributing to the regulation of adipocyte function by increasing or decreasing its protein levels. Thus, future research should focus on screening for the effectors of OS-9 in adipocytes and the investigation of the precise mechanism by which OS-9 regulates them. Determination of the complete mechanism requires further investigation. 


\section{Acknowledgements}

This study was supported by the National Basic Research Program of China (grant no. 2007CB914401), the Ministry of Science and Technology of China and the Scholarship Award for Excellent Doctoral Student granted by the Ministry of Education of China. The authors thank Dr Peng Li and the members of Dr Li's laboratory at Tsinghua University for the technical assistance and helpful discussions.

\section{References}

1. Armani A, Mammi C, Marzolla V, et al: Cellular models for understanding adipogenesis, adipose dysfunction, and obesity. J Cell Biochem 110: 564-572, 2010.

2. Ozcan U, Cao Q, Yilmaz E, et al: Endoplasmic reticulum stress links obesity, insulin action, and type 2 diabetes. Science 306: 457-461, 2004

3. Nakatani Y, Kaneto H, Kawamori D, et al: Involvement of endoplasmic reticulum stress in insulin resistance and diabetes. J Biol Chem 280: 847-851, 2005.

4. Hirosumi J, Tuncman G, Chang L, et al: A central role for JNK in obesity and insulin resistance. Nature 420: 333-336, 2002.

5. Sabio G, Das M, Mora A, et al: A stress signaling pathway in adipose tissue regulates hepatic insulin resistance. Science 322: $1539-1543,2008$

6. Kim W, Spear ED and Ng DT: Yos9p detects and targets misfolded glycoproteins for ER-associated degradation. Mol Cell 19: 753-764, 2005.

7. Szathmary R, Bielmann R, Nita-Lazar M, Burda $P$ and Jakob CA: Yos9 protein is essential for degradation of misfolded glycoproteins and may function as lectin in ERAD. Mol Cell 19: 765-775, 2005.

8. Denic V, Quan EM and Weissman JS: A luminal surveillance complex that selects misfolded glycoproteins for ER-associated degradation. Cell 126: 349-359, 2006.

9. Carvalho P, Goder V and Rapoport TA: Distinct ubiquitin-ligase complexes define convergent pathways for the degradation of ER proteins. Cell 126: 361-373, 2006.

10. Gauss R, Jarosch E, Sommer T and Hirsch C: A complex of Yos9p and the HRD ligase integrates endoplasmic reticulum quality control into the degradation machinery. Nat Cell Biol 8: 849-854, 2006.

11. Su YA, Hutter CM, Trent JM and Meltzer PS: Complete sequence analysis of a gene (OS-9) ubiquitously expressed in human tissues and amplified in sarcomas. Mol Carcinog 15: 270-275, 1996.
12. Kimura $\mathrm{Y}$, Nakazawa $\mathrm{M}$ and Yamada $\mathrm{M}$ : Cloning and characterization of three isoforms of OS-9 cDNA and expression of the OS-9 gene in various human tumor cell lines. J Biochem 123: 876-882, 1998

13. Christianson JC, Shaler TA, Tyler RE and Kopito RR: OS-9 and GRP94 deliver mutant alpha1-antitrypsin to the Hrd1-SEL1L ubiquitin ligase complex for ERAD. Nat Cell Biol 10: 272-282, 2008.

14. Baek JH, Mahon PC, Oh J, et al: OS-9 interacts with hypoxiainducible factor lalpha and prolyl hydroxylases to promote oxygen-dependent degradation of HIF-1alpha. Mol Cell 17: 503-512, 2005.

15. Mikami K, Yamaguchi D, Tateno $\mathrm{H}$, et al: The sugar-binding ability of human OS-9 and its involvement in ER-associated degradation. Glycobiology 20: 310-321, 2010.

16. Hosokawa N, Kamiya Y, Kamiya D, Kato K and Nagata K: Human OS-9, a lectin required for glycoprotein endoplasmic reticulum-associated degradation, recognizes mannose-trimmed N-glycans. J Biol Chem 284: 17061-17068, 2009.

17. Meusser B, Hirsch C, Jarosch E and Sommer T: ERAD: the long road to destruction. Nat Cell Biol 7: 766-772, 2005.

18. Alcock F and Swanton E: Mammalian OS-9 is upregulated in response to endoplasmic reticulum stress and facilitates ubiquitination of misfolded glycoproteins. J Mol Biol 385: 1032-1042, 2009.

19. Qi J, Gong J, Zhao T, et al: Downregulation of AMP-activated protein kinase by Cidea-mediated ubiquitination and degradation in brown adipose tissue. EMBO J 27: 1537-1548, 2008.

20. Dull T, Zufferey R, Kelly M, et al: A third-generation lentivirus vector with a conditional packaging system. J Virol 72: 8463-8471, 1998.

21. Nian Z, Sun Z, Yu L, Toh SY, Sang J and Li P: Fat-specific protein 27 undergoes ubiquitin-dependent degradation regulated by triacylglycerol synthesis and lipid droplet formation. J Biol Chem 285: 9604-9615, 2010.

22. Toh SY, Gong J, Du G, et al: Up-regulation of mitochondrial activity and acquirement of brown adipose tissue-like property in the white adipose tissue of fsp27 deficient mice. PLoS ONE 3: e2890, 2008.

23. Joost HG and Schurmann A: Subcellular fractionation of adipocytes and 3T3-L1 cells. Methods Mol Biol 155: 77-82, 2001.

24. Storch J and Thumser AE: The fatty acid transport function of fatty acid-binding proteins. Biochim Biophys Acta 1486: 28-44, 2000.

25. Wang Y, Fu X, Gaiser S, et al: OS-9 regulates the transit and polyubiquitination of TRPV4 in the endoplasmic reticulum. J Biol Chem 282: 36561-36570, 2007. 